\title{
QCD factorisation breaking in diffractive gauge bosons production at the LHC
}

\author{
Roman Pasechnik* \\ Department of Astronomy and Theoretical Physics, Lund University, SE 223-62 Lund, Sweden \\ E-mail: Roman.Pasechnikethep.Iu.se
}

\section{Boris Kopeliovich and Irina Potashnikova}

Departamento de Física Universidad Técnica Federico Santa María; and Instituto de Estudios Avanzados en Ciencias e Ingeniería; and

Centro Científico-Tecnológico de Valparaíso;

Casilla 110-V, Valparaíso, Chile

We discuss single diffractive gauge bosons $\left(\gamma^{*}, W^{ \pm}, Z\right)$ production in proton-proton collisions at LHC energy $(14 \mathrm{TeV})$ in the framework of the color dipole approach. The calculations are performed for gauge bosons produced at forward rapidities. The diffractive cross section is predicted as a function of fractional momentum and invariant mass of the lepton pair. We found a dramatic breakdown of the diffractive QCD factorisation caused by an interplay of hard and soft interactions.

Sixth International Conference on Quarks and Nuclear Physics,

April 16-20, 2012

Ecole Polytechnique, Palaiseau, Paris

\footnotetext{
* Speaker.
} 


\section{Sources of violation of the diffractive factorisation}

The main difficulty in the formulation of a theoretical QCD-based framework for diffractive scattering arises from the fact that it is essentially contaminated by soft, non-perturbative interactions. For example, diffractive deep-inelastic scattering (DIS), $\gamma^{*} p \rightarrow X p$, although it is a higher twist process, is dominated by soft interactions.

Within the dipole approach [W] such a process looks like elastic scattering of $\bar{q} q$ dipoles of different sizes, and of higher Fock states containing more partons. Although formally the process $\gamma^{*} \rightarrow X$ is an off-diagonal diffraction, it does not vanish in the limit of unitarity saturation, the so called black disc limit. This happens because the photon distribution functions and hadronic wave functions are not orthogonal. Such a principal difference between diffractive processes in DIS and hadronic collisions is one of the reasons for breakdown of diffractive QCD factorization based e.g. on the Ingelman-Schlein model [[]]. The phenomenological models based on assumptions of the diffractive factorisation, which are widely discussed in the literature (see e.g. Ref. [3]), predict a significant increase of the ratio of the diffractive to inclusive gauge bosons production cross sections with energy. This will be tested soon at the LHC.

The process under discussion, diffractive abelian radiation of electroweak gauge bosons, is the real off-diagonal diffraction. It vanishes in the black-disc limit, and may be strongly suppressed by the absorptive corrections even being far from the unitarity bound. The suppression caused by the absorptive corrections, also known as the survival probability of a large rapidity gap, is related to the initial and final state interactions. Usually the survival probability is introduced in the diffractive cross section in a probabilistic way and is estimated in oversimplified models, like eikonal, quasi-eikonal, two-channel approximations, etc. The advantage of the dipole approach is the possibility to calculate directly (although in a model-dependent way) the full diffractive amplitude, which contains all the absorption corrections, because it employs the phenomenological dipole cross section fitted to data.

Another source of factorization breaking is the simple observation that diffractive abelian radiation by a quark vanishes in the forward direction (zero momentum transfer to the target) [四]. Indeed, the Fock components of the quark with or without the abelian boson $\left(\gamma^{*}, Z, W\right.$, Higgs boson) interact with the same total cross sections, because only the quark interacts strongly. Therefore, after integration of the amplitude over impact parameter the Fock state decomposition of the projectile remains unchanged, and only elastic $q p$ scattering is possible.

In the case of $p p$ collisions the directions of propagation of the proton and its quarks do not coincide. Already this is sufficient to get a nonvanishing diffractive abelian radiation in forward scattering. Moreover, interaction with the spectator partons opens new possibilities for diffractive scattering, namely the color exchange in interaction of one projectile parton, can be compensated (neutralized) by interaction of another projectile parton []. It was found in Ref. [6] that this contribution leads to a dominant contribution to the diffractive abelian radiation in the forward direction. This mechanism, leading to a dramatic violation of diffractive QCD factorisation, is under consideration in the present paper. 


\section{Single diffractive cross section}

Let us assume that the gauge boson is emitted by the quark $q_{1}$. As a result of the hard emission the quark position in the impact parameters, being initially $\vec{r}_{1}$, gets shifted to $\vec{r}_{1}+\alpha \vec{r}$. Applying the completeness relation to the wave function of the proton remnant in the final state, we get the diffractive $G^{*}$ production cross section in the following form [目]

$$
\begin{aligned}
\frac{d^{5} \sigma_{\lambda_{G}}(p p}{d^{2} q_{\perp}} d & \left.\rightarrow p x_{1} d^{2} \sigma_{\perp}^{*} X\right) \\
& \times \Psi_{V-A}^{\lambda_{G}}(\vec{r}, \alpha, M) \Psi_{V-A}^{\lambda_{G} *}\left(\vec{r}^{\prime}, \alpha, M\right)\left|\Psi_{i}\left(\vec{r}_{1}, \vec{r}_{2}, \vec{r}_{3} ; x_{q},\left\{x_{q}^{2,3, \ldots}\right\},\left\{x_{g}^{2,3, \ldots}\right\}\right)\right|^{2} \\
& \times \Delta\left(\vec{r}_{1}, \vec{r}_{2}, \vec{r}_{3} ; \vec{b} ; \vec{r}, \alpha\right) \Delta\left(\vec{r}_{1}, \vec{r}_{2}, \vec{r}_{3} ; \vec{b}^{\prime} ; \vec{r}^{\prime}, \alpha\right) e^{i \vec{\delta}_{\perp} \cdot\left(\vec{b}-\vec{b}^{\prime}\right)} e^{i \vec{l}_{\perp} \cdot \alpha\left(\vec{r}-\vec{r}^{\prime}\right)}
\end{aligned}
$$

where $\vec{r}_{i}, x_{q / g}^{i}$ are the transverse coordinates and fractional light-cone momenta of the valence/sea quarks and gluons, $\Psi_{i}$ is the proton wave function, the summation is performed over all valence/sea quarks and gluons in the proton, and the light-cone fraction of the quark emitting the gauge boson $x_{q}^{1} \equiv x_{q}$ is fixed by the external phase space variables $x_{1}$ and $\alpha$ due to the momentum conservation, namely, $x_{q}=x_{1} / \alpha, x_{1}=q^{+} / P_{1}^{+}$, where $P_{1}$ is the 4-momentum of the projectile proton, $q$ is the 4-momentum of the produced gauge boson, and

$\Delta=-2 \operatorname{Im} f_{e l}\left(\vec{b}, \vec{r}_{1}-\vec{r}_{2}\right)+2 \operatorname{Im} f_{e l}\left(\vec{b}, \vec{r}_{1}-\vec{r}_{2}+\alpha \vec{r}\right)-2 \operatorname{Im} f_{e l}\left(\vec{b}, \vec{r}_{1}-\vec{r}_{3}\right)+2 \operatorname{Im} f_{e l}\left(\vec{b}, \vec{r}_{1}-\vec{r}_{3}+\alpha \vec{r}\right)$,

is the properly normalized diffractive amplitude, where $f_{e l}\left(\vec{b}, \vec{r}_{1}-\vec{r}_{2}\right)$ is the partial elastic amplitude for dipole of transverse size $r$ colliding with a proton at impact parameter $b$ to be specified below. As expected, the diffractive amplitude $\Delta$ is proportional to the difference between elastic amplitudes for the dipoles of slightly different sizes. This difference is suppressed by absorptive corrections, the effect sometimes called survival probability of large rapidity gaps.

The amplitude Eq. ([D) is the full expression, which includes by default the effect of absorption and does not need any extra survival probability factor. This can be illustrated on a simple example of elastic dipole scattering off a potential. The dipole elastic amplitude has the eikonal form,

$$
\operatorname{Im} f_{e l}\left(\vec{b}, \vec{r}_{1}-\vec{r}_{2}\right)=1-\exp \left[i \chi\left(\vec{r}_{1}\right)-i \chi\left(\vec{r}_{2}\right)\right], \quad \chi(b)=-\int_{-\infty}^{\infty} d z V(\vec{b}, z),
$$

and $V(\vec{b}, z)$ is the potential, which depends on the impact parameter and longitudinal coordinate, and is nearly imaginary at high energies. The difference between elastic amplitudes with a shifted quark position, which enters the diffractive amplitude, reads,

$$
\operatorname{Im} f_{e l}\left(\vec{b}, \vec{r}_{1}-\vec{r}_{2}+\alpha \vec{r}\right)-\operatorname{Im} f_{e l}\left(\vec{b}, \vec{r}_{1}-\vec{r}_{2}\right)=\exp \left[i \chi\left(\vec{r}_{1}\right)-i \chi\left(\vec{r}_{2}\right)\right] \exp \left[i \alpha \vec{r} \cdot \vec{\nabla} \chi\left(\vec{r}_{1}\right)\right] .
$$

The first factor $\exp \left[i \chi\left(\vec{r}_{1}\right)-i \chi\left(\vec{r}_{2}\right)\right]$ is exactly the survival probability amplitude, which vanishes in the black disc limit, as it should be. This proves that the cross section Eq. (D. Includes the effect of absorption. Notice that usually the survival probability factor is introduced into the diffractive cross section probabilistically, while in Eq. (‥D) it is treated quantum-mechanically, at the amplitude level. 
All the elastic amplitudes in Eq. (ل) implicitly depend on energy. They cannot be calculated reliably, but but are known from phenomenology. Since large dipole sizes $\left|\vec{r}_{i}-\vec{r}_{j}\right| \sim b \sim R_{p}$, $i \neq j$ ( $R_{p}$ is the mean proton size) are important in Eq. (2.]), the Bjorken variable $x$ is ill defined, and the collisions energy is a more appropriate variable. A parametrization of the dipole cross section as function of $s$ was proposed and fitted to data in Ref. [四], and the corresponding partial dipole amplitude is given by e.g. Ref. [प]. For more details on the Color Dipole formalism for the diffractive gauge bosons production and consequences, see Ref. [ $[8]$.

Finally, we parameterize the proton wave function assuming the symmetric Gaussian shape for the spacial valence quark distributions in the proton, as

$$
\begin{aligned}
\mid \Psi_{i}\left(\vec{r}_{1}, \vec{r}_{2}, \vec{r}_{3} ; x_{q},\left\{x_{q}^{2,3, \ldots}\right\},\left.\left\{x_{g}^{2,3, \ldots}\right)\right|^{2}\right. & =\frac{3 a^{2}}{\pi^{2}} e^{-a\left(r_{1}^{2}+r_{2}^{2}+r_{3}^{2}\right)} \rho\left(x_{q},\left\{x_{q}^{2,3, \ldots}\right\},\left\{x_{g}^{2,3, \ldots}\right\}\right) \\
& \times \delta\left(\vec{r}_{1}+\vec{r}_{2}+\vec{r}_{3}\right) \delta\left(1-x_{q}-\sum_{j} x_{q / g}^{j}\right)
\end{aligned}
$$

where sum is taken over all valence/sea quarks and gluons not participating in the hard interaction, $x_{q}$ is defined above, $a=\left\langle r_{c h}^{2}\right\rangle^{-1}$ is the inverse proton mean charge radius squared; $\rho$ is the valence quark distribution function in the proton. Integrating over the fractional momenta of all partons not participating in the hard interaction we arrive at the single valence quark distribution in the proton, probed by the hard process - radiation of a heavy gauge boson,

$$
\int \prod_{i} d x_{q}^{i} d x_{g}^{i} \delta\left(1-x_{q}-\sum_{j} x_{q / g}^{j}\right) \rho\left(x_{q},\left\{x_{q}^{2,3, \ldots}\right\},\left\{x_{g}^{2,3, \ldots}\right\}\right)=\rho_{q}\left(x_{q}\right),
$$

where $q$ denotes the quark flavor emitting the gauge boson $G$ with the fraction $x_{q}$. In the case of diffractive Drell-Yan reaction [焑], generalization of the three-body proton wave function (2.4) including different quark and antiquark flavors leads to the proton structure function as,

$$
\sum_{q} Z_{q}^{2}\left[\rho_{q}\left(x_{q}\right)+\rho_{\bar{q}}\left(x_{q}\right)\right]=\frac{1}{x_{q}} F_{2}\left(x_{q}\right) .
$$

However, in the case of diffractive $W$ and $Z$ production the coupling factor $\mathscr{C}_{q}^{G} g_{v / a, q}^{G}$ varies for different (valence/sea) quark species in the proton, so one has to deal with the original quark densities. Similar to the diffractive DY case, in actual numerical calculations below, when summing up the contributions of different quark flavors, we will generalize the above approach including the sea quark and antiquark densities in the proton at the hard scale imposed by the mass of the gauge boson.

\section{Numerical results}

We now turn to a discussion of the numerical results for the most important observables. First of all, we are interested in the di-lepton (à la Drell-Yan pair) production channel as the simplest one. Although quark production channel could also be of interest, this case will be considered elsewhere. In Fig. W for LHC energy $\sqrt{s}=14 \mathrm{TeV}$ we present the single diffractive cross sections for $Z^{0}, \gamma^{*}$ (diffractive DY) and $W^{ \pm}$bosons production, differential in the di-lepton mass squared $d \sigma_{s d} / d M^{2}$ (left panels) and its longitudinal momentum fraction, $d \sigma_{s d} / d x_{1}$ (right panels). These plots do not 
reflect particular detector constraints - a thorough analysis including detector acceptances and cuts has to be done separately. The $M^{2}$ distributions here are integrated over the ad hoc interval of fractional boson momentum $0.3<x_{1}<1$, corresponding to the forward rapidity region (at not extremely large masses). Then the mass distribution is integrated over the potentially interesting invariant mass interval $5<M^{2}<10^{5} \mathrm{GeV}^{2}$, and can be easily converted into (pseudo)rapidity ones widely used in experimental studies, if necessary.
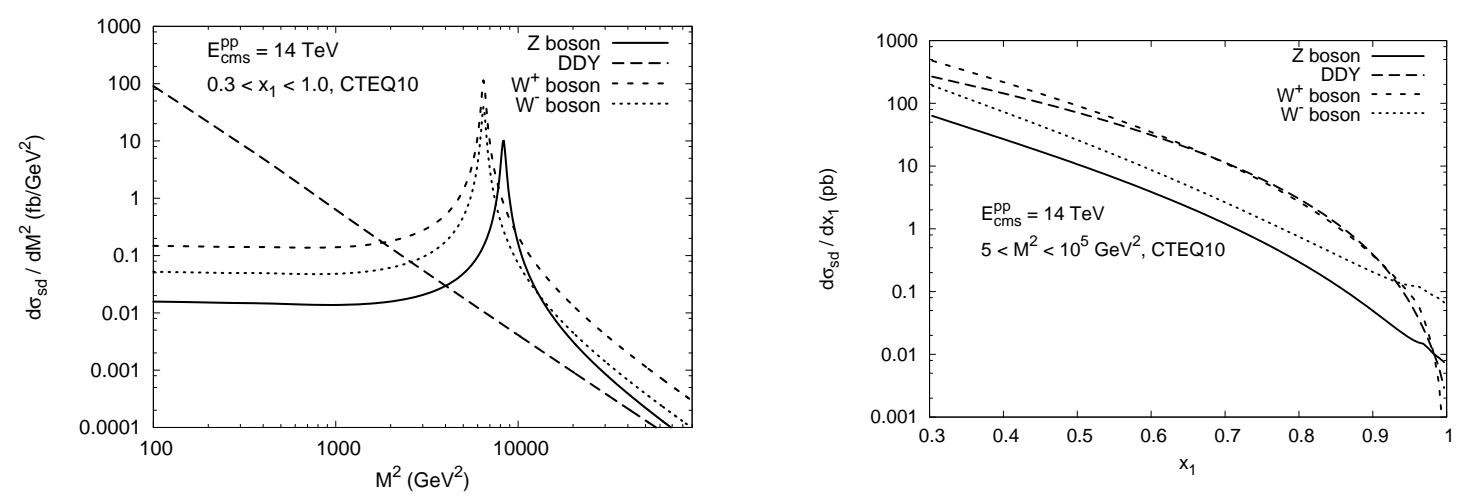

Figure 1: Diffractive gauge boson production cross section as function of di-lepton invariant mass squared $M^{2}$ (left panel) and boson fractional light-cone momentum $x_{1}$ (right panel) in $p p$ collisions at the LHC energy $\sqrt{s}=14 \mathrm{GeV}$.
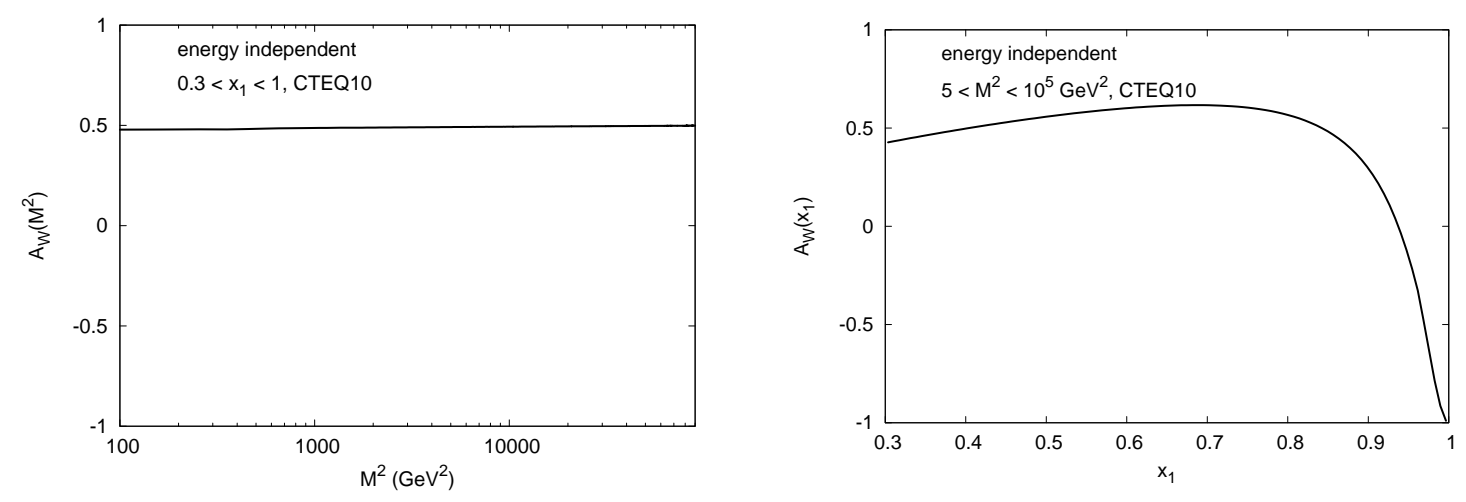

Figure 2: Charge asymmetry in the single diffractive $W^{+}$and $W^{-}$cross sections as a function of $M^{2}$, at fixed $x_{1}=0.5$ (left panel), and $x_{1}$, at fixed $M^{2}=M_{W}^{2}$ (right panel) at the LHC energy $\sqrt{s}=14 \mathrm{TeV}$.

The $M^{2}$ distributions of the $Z^{0}$ and $W^{ \pm}$bosons clearly demonstrate their resonant behavior, and in the resonant region significantly exceed the corresponding diffractive Drell-Yan component; only for very low masses the $\gamma^{*}$ contribution becomes important (left panels). For $x_{1}$ distribution, when integrated over low mass and resonant regions, diffractive $W^{+}$and $\gamma^{*}$ components become comparable to each other, both in shapes and values, whereas the $W^{-}$and, especially, $Z$-boson production cross section are noticeably lower (right panels). Quite naturally, the $W^{-}$cross section is (in analogy with the well-known inclusive $W^{ \pm}$production) smaller than the $W^{+}$one due to differences in valence $u$ - and $d$-quark densities (dominating over sea quarks at large $x_{q}$ ) in the proton, the bosons couple to. So the precise measurement of differences in forward diffractive 
$W^{+}$and $W^{-}$rates would allow to constrain quark content of the proton at large $x_{q} \equiv x_{1} / \alpha$. In W, and in all calculation below we have used the most recent CTEQ10 valence/sea quark PDFs parametrization [Q], if not declared otherwise.

As one of the important observables, sensitive to the difference between $u$ - and $d$-quark PDFs, the $W^{ \pm}$charge asymmetry $A_{W}$ is shown in Fig. $\square$ differentially as a function of the di-lepton invariant mass squared $M^{2}$ and integrated over $0.3<x_{1}<1.0$ interval (left panel) and as a function of the boson momentum fraction $x_{1}$ and integrated over $5<M^{2}<10^{5} \mathrm{GeV}^{2}$ interval (right panel). The asymmetry turns out to be independent on both the hard scale $M^{2}$ and the c.m. energy $\sqrt{s}$. One concludes that, due to different $x$-shapes of valence $u, d$ quark PDFs, at smaller $x_{1} \lesssim 0.9$ the diffractive $W^{+}$bosons' rate dominates over $W^{-}$one. However, at large $x_{1} \rightarrow 1$ the $W^{-}$boson cross section becomes increasingly important and strongly dominates over the $W^{+}$one.

\section{Conclusions}

In opposite to the diffractive Deep-Inelastic-Scattering (DDIS), the single diffractive gauge bosons production cross section behaves as $\sim \vec{r} \cdot \vec{R}$, soft and hard fluctuations contribute in this process on the same footing, and their interplay does not depend on the hard scale, similar to the inclusive gauge bosons production. Hence, the forward diffractive abelian radiation turns out to be of the leading twist nature, and the diffractive-to-inclusive production cross sections ratio can depend on the hard scale only weakly through the $x$-dependence of the saturation scale, or more precisely $R_{0}\left(x_{2}\right)$, and can only increase.

The fundamental interplay between the hard and soft interactions in the forward diffractive Abelian radiation is the major reason for the diffractive $Q C D$ factorisation breaking leading to quite unusual features of the corresponding observables (for a similar discussion in the diffractive DY, see Ref. [可]). As we have emphasized above, this interplay is absent in the DDIS and in diffractive QCD factorisation-based approaches to the diffractive DY (see e.g. Ref. [ए]]) leading to the energy and scale dependence of the corresponding cross section which is completely opposite to the one predicted above by the Color Dipole model (for more details, see Ref. [[]]).

\section{References}

[1] B. Z. Kopeliovich, L. I. Lapidus and A. B. Zamolodchikov, JETP Lett. 33, 595 (1981).

[2] G. Ingelman, P. E. Schlein, Phys. Lett. B152 256 (1985).

[3] M. B. Gay Ducati, M. M. Machado and M. V. T. Machado, Phys. Rev. D 75, 114013 (2007).

[4] B. Z. Kopeliovich, A. Schäfer and A. V. Tarasov, Phys. Rev. D62, 054022 (2000).

[5] R. Pasechnik, R. Enberg and G. Ingelman, Phys. Rev. D 82, 054036 (2010); Phys. Lett. B 695, 189 (2011).

[6] R. S. Pasechnik and B. Z. Kopeliovich, arXiv:1109.6690 [hep-ph]; Eur. Phys. J. C 71, 1827 (2011).

[7] B. Z. Kopeliovich, H. J. Pirner, A. H. Rezaeian and I. Schmidt, Phys. Rev. D 77, 034011 (2008).

[8] R. Pasechnik, B. Kopeliovich and I. Potashnikova, arXiv:1204.6477 [hep-ph].

[9] H. L. Lai et al, Phys. Rev. D 82, 074024 (2010).

[10] G. Kubasiak and A. Szczurek, Phys. Rev. D 84, 014005 (2011). 